\title{
Interventions for paediatric surgery patients with comorbid autism spectrum disorder: a systematic literature review
}

\author{
Scott Koski, Robin L Gabriels, Carol Beresford
}

University of Colorado Anschutz Medical Campus and the Children's Hospital Colorado, Aurora, Colorado, USA

\section{Correspondence to} Dr Scott Koski, Department of Psychiatry, University of Colorado Anschutz Medical Campus/Children's Hospital Colorado, 13123 E 16th Ave, B130, Aurora, CO 80045 , USA; scott.koski@ucdenver.edu

Received 3 March 2016 Revised 27 April 2016 Accepted 9 May 2016 Published Online First 25 May 2016

\section{SLinked}

- http://dx.doi.org/10.1136/ archdischild-2016-311299

\section{CrossMark}

To cite: Koski $S$,

Gabriels RL, Beresford C.

Arch Dis Child

2016;101:1090-1094.

\section{ABSTRACT}

Aims To survey perioperative management practices for paediatric patients diagnosed with autism spectrum disorder (ASD).

Methods A systematic review was carried out of empirical studies and case reports published in peerreviewed journals of current best practices and behavioural interventions for paediatric patients with ASD who had undergone surgery.

Results The final sample included 11 articles published between 1997 and 2016 that met broad inclusion criteria of surveying perioperative interventions for the ASD population. There is broad endorsement across the scant publications on this topic of the following practices: increased attention to individual patient needs, rehearsal and other desensitisation efforts, departure from a sole focus on sedation or restraint of the combative or uncooperative patient and engaging caregivers in tuning perioperative management to individual needs.

Conclusions This review supports the need for an individualised structure and approach to the perioperative care of these unique patients.

\section{INTRODUCTION}

For the typical paediatric patient presenting for surgery, a deluge of new faces, sounds, smells, icebox-like room temperatures and other sensory novelties can be quite overwhelming for all and particularly for the patient diagnosed with autism spectrum disorder (ASD). The diagnosis of ASD is based on core behavioural deficits in social interactions, communications, and the co-occurrence of restricted, repetitive and stereotyped behaviours and interests. ${ }^{1}$ For example, insistence on 'sameness' is a common feature of ASD and can be expressed as a significant behavioural struggle when routines are interrupted and when these patients are placed in unfamiliar environments, particularly those that involve separation from caregivers. ${ }^{2}$ Problems of coexisting psychiatric, medical, intellectual ability, language and/or sensory regulation diagnoses can increase the complexity of behavioural management. For example, challenging behaviours can range from hyperactivity, obsessive-compulsive rigidities, self-injury, aggression, stereotypies, tics and a range of affective symptoms. ${ }^{3}$

Behavioural challenges in the ASD population are common in perioperative settings and require careful consideration of the wide variety of profiles of these patients. ${ }^{4}$ Kopecky et $a l^{4}$ found that in 80 surveys completed by parents of children (average

\section{What is already known on this topic?}

- Much of the early perioperative literature on patients with ASD focuses on the 'combative' or 'uncooperative' patient and less on the root causes of the behaviours which lead to such labels.

- Historically, the literature concerning perioperative management of this population has been dominated by anaesthesia, examining the risks/benefits of various sedation protocols.

- As noted in this review, apart from a small handful of visionary clinicians (ie, Seid et al, ${ }^{10}$ van der Walt and Moran ${ }^{13}$ ) attention to behaviourally informed interventions has been rare.

\section{What this study adds?}

- A growing number (albeit still small) of hospital-based teams have undertaken new behavioural approaches in the perioperative setting and achieved improved clinical outcomes.

- This review aims at uncovering and assimilating such practices and mining them for generalisable wisdom. The individualisation theme noted herein is one such pearl.

age 14.6 years) with ASD, who were mostly patients at Massachusetts General Hospital, 38\% of patients with ASD expressed their needs or desires using sign language or gestures and 31\% used communication tools, including picture exchange communication systems or an electronic device; only 23\% expressed their needs verbally. Mills highlights the role of Social Stories (developed by Carol Gray) which recognise that children with ASD often struggle to discern, anticipate and understand the flow and basic facts of social situations. ${ }^{5}$ Social Stories can facilitate successful interactions by diminishing the element of surprise through the use of visual and other supports to desensitise a child/adolescent patient with ASD to new elements of a procedure or examination.

Much of the early perioperative literature on the ASD population focuses on the 'combative' or 'uncooperative' patient and less on the root causes 
of the behaviours which lead to such labels. Hospital-based teams have undertaken this challenge and implemented new behavioural approaches that have resulted in observed improvements in clinical outcomes. ${ }^{267}$ However, empirical evidence of the utility of specific perioperative management strategies is limited, leaving providers to rely on anecdotal evidence. The principal aim of this review was to survey perioperative management practices for children with ASD.

\section{METHODS}

\section{Search strategy}

Studies were identified by searching electronic databases (ie, OVID SR, Medline, PsycINFO, AMED, PubMed and Google Scholar) between 1990 and 2016. Search terms for all databases included at least one identifier for ASD and at least one identifier for perioperative intervention in the full text of the article. Specifically, identifiers included 'ASD' OR 'autism' OR 'autistic' OR 'Asperger's' OR 'pervasive developmental disorder'. Terms for perioperative intervention included 'perioperative' OR 'surgery' OR 'perioperative management' OR 'behavioural intervention'.

\section{Study selection}

Publications were included in this review if they were:

- published in a peer-reviewed journal;

- contained original, empirical and/or case report information, focusing on perioperative interventions for paediatric patients with ASD intended to mitigate or ameliorate emotional/behavioural dysregulation with or without drug treatment.

Publications were excluded if there was an exclusive focus on surgical interventions (eg, neurosurgery for patients with comorbid epilepsy) or if there was a primary focus on sedation protocols in the perioperative setting.

\section{Data extraction and evaluation}

Information was extracted from each study or case report to achieve the principal aim of surveying the perioperative management of the ASD population. Study participants were often parents and/or caregivers. Data also included study results and/ or guiding principles obtained from interventions used. Study identification data were extracted, including first author, publication year, country of corresponding author and journal name.

\section{RESULTS}

This literature search resulted in 66 citations. Fifty-five were excluded for reasons noted in the 'Methods' section. The final sample included 11 articles published between 1997 and 2016 that met the inclusion criteria (see figure 1). Despite narrowing the focus to English-language articles only, international researchers were broadly represented. Articles were published in a variety of journals, including those dealing with paediatric anaesthesia, paediatric oto-rhino-laryngology and paediatric nursing. Study designs, participants, interventions and outcome measures varied widely. In view of this heterogeneity, the results of this review focus on a descriptive and qualitative synthesis rather than a meta-analysis.

Across the 11 articles surveyed no standardised terminology emerged to describe the perioperative experience. Rather, a variety of terms were used, including in-hospital management, needs of hospitalised patients with ASD, improving management of patients with ASD having scheduled surgery, improving psychiatric hospital care for paediatric patients with ASD, induction of anaesthesia in a combative child, perioperative psychosocial interventions for autistic children, experiences of parents with their child's anaesthetics, an audit of perioperative management of autistic children, caring for children and adolescents with ASD who require challenging procedures, all of which have descriptive value. None used this review's terminology-'perioperative interventions for ASD'.

Selected studies/case reports are summarised by study characteristics, methods and outcomes in table 1. It is important to note that most of the articles selected were case reports. Only three studies included a large sample. The most common study designs were case reports and clinical case series. Many of the case reports were retrospective reviews of a particular perioperative intervention and its ability to enhance communication or behavioural outcomes. Several studies used survey data, principally with caregivers of patients with ASD, to assess the child's experience.

Articles varied by outcome measures, including quantitative and qualitative data derived from investigator-designed surveys and open-ended or structured interviews primarily with caregivers.

All articles suggested some form of 'best practice' derived from their particular interventions as summarised in table 1 . Key structures identified by the articles reviewed to proactively facilitate a successful perioperative experience for the patient are as follows: (1) collaborating with the caregiver to gather critical information about the patient's specific needs and preferences in preparation for surgery; (2) identifying a process for communicating information obtained from the caregiver to the perioperative team, appreciating the complexity of bridging these contrasting cultures, and including caregivers in the perioperative setting as 'real-time' interpreters of patients' needs; (3) making environmental modifications to the perioperative setting as needed to match patients' specific needs and preferences. For example, including a service dog to create a calm association for the patient when introducing them to the perioperative setting, ${ }^{12}$ providing opportunities for the patient to become familiar with the setting (including staff) and expectations before surgery and finally, providing the patient with choices and distractions.

\section{DISCUSSION}

This review surveyed the literature to identify perioperative practices specifically tailored to paediatric patients with ASD. A systematic literature search resulted in only 11 articles that met broad inclusion criteria of empirically evaluating perioperative interventions for this population. Most used simple pre-post intervention designs or were case reports or series. No common perception of the unique needs of this population or ways to understand the root cause(s) of problem behaviours that may occur in the preoperative setting were found. Additionally, information about concurrent treatments and medications was limited; this absence of information is a limitation of this study as both treatment and medications may influence the effectiveness of any perioperative intervention. Most 'best practice' suggestions were derived from interviews with caregivers and direct patient observation. Three common themes emerged: (1) collaborating with the caregiver to inform patient management, (2) developing a process for communicating the information gathered from caregivers to perioperative staff and (3) modifying the perioperative environment, based on patient-specific needs gathered from the caregiver. 
Figure 1 PRISMA statement.

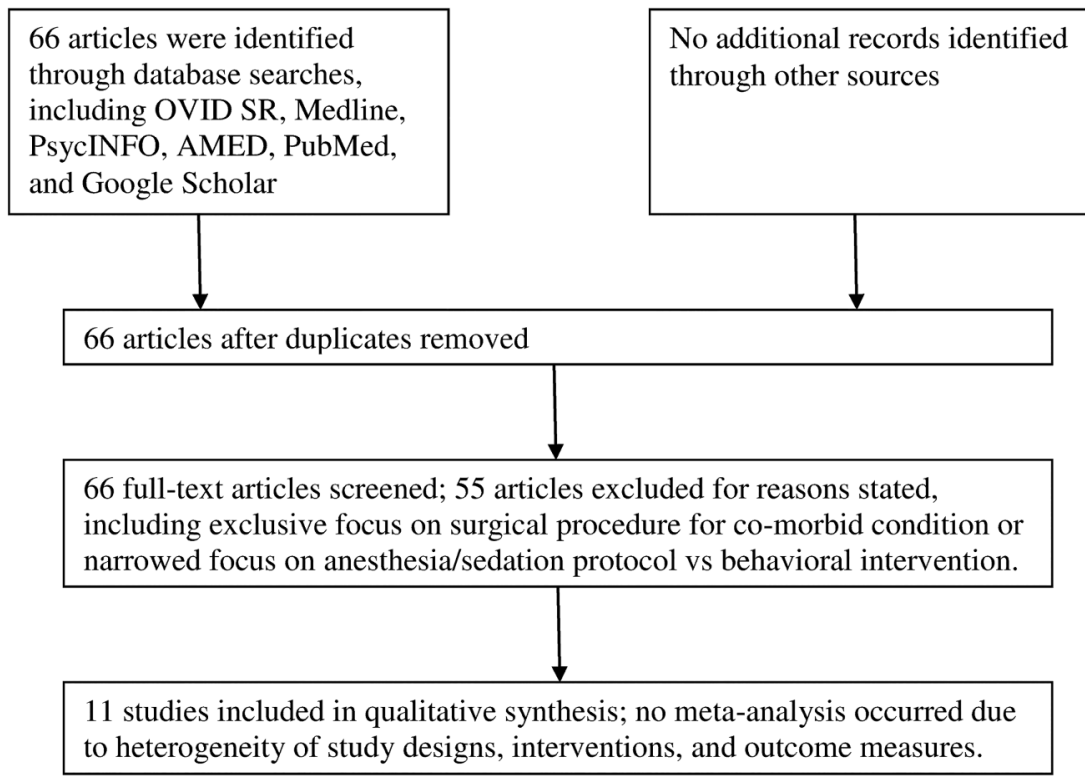

\section{Collaborating with the caregiver to inform patient management}

This review highlights 'individualisation of care' as a pragmatic response to years of effort to make this patient population conform to established perioperative routines. It emphasises the practicality of setting aside well-rehearsed pre- and postoperative approaches to allow for a tailored response to this unique set of patients. As the previous section underlines, parents/caregivers are almost always the main people to shape individualisation of perioperative support to allow for an effective care plan. Extrapolation suggests attention to individualisation may also smooth perioperative transitions. Souders $e t a l^{14}$ and Thompson and Tielsch-Goddard ${ }^{8}$ both used the severity of ASD to inform individualisation of perioperative intervention. Thompson and Tielsch-Goddard's ${ }^{8}$ quality improvement project for surgical admissions of children with ASD provides a template for individualisation of care. In Thompson's follow-up interviews, parents stated their appreciation of the individualised care and nurses reported their increased satisfaction in caring for patients with ASD when additional attention is given to preventive stress management by inclusion of parents' and children's detailed knowledge of the unique needs. Individualised behavioural plans and behavioural/communication strategies enhance the perioperative experience of these patients. The problem of replication and generalisation of any specific behavioural or structural approach remains complex when individualisation is a primary element of every effective patient management strategy.

\section{Developing a process for communicating information gathered from caregivers to perioperative staff}

Thompson and Tielsch-Goddard ${ }^{8}$ underlined the significance of better communication of individualised needs, pragmatic assessment of communication strategies and sensory differences unique to each individual and learning from parents, allowing their accumulated wisdom to guide interventions. To facilitate transmission of usable information gathered from caregivers, Thompson's group developed a 'Hand-off Communication Tool' that was available to every member of the perioperative team to provide awareness of specific needs, and also to allow information to be added in real-time about staff interventions and patient responses. With this dynamic approach, only two of 43 patients had a total loss of behavioural control without resolution and both occurred postoperatively with parents at the bedside. $^{8}$

Modifying the perioperative environment-based patient-specific needs gathered from the caregiver

Seid et $a l^{10}$ were visionary in delineating the perioperative psychosocial elements for children with ASD undergoing ear, nose and throat surgery. Lindberg et $a l^{11}$ chose to focus on the experiences of the caregivers, illustrating how continual liaison with caregivers was strategic in providing alternatives to unnecessary suffering for the patient. Lindberg et $a l^{11}$ eloquently describe the importance of adapting the environment to meet patients' unique needs. van der Walt and Moran ${ }^{13}$ and Seid et $a l^{10}$ emphasised the importance of including caregivers in vigorous preplanning of the periprocedural details, as well as involvement from induction to the early recovery phase. Flexible admission, which enabled a decreased waiting time, was another important finding. ${ }^{13}$

\section{CONCLUSIONS}

In summary, this review revealed a paucity of research in this particular area of hospital care for children and adolescent patients with ASD. However, included studies helped to lay the foundation of an emerging body of evidence. The dearth of high-quality research might have led to biased conclusions from weak evidence. Thus, findings in the current review should not be interpreted as evidence for a particular perioperative intervention for ASD. Instead, they offer preliminary support for the concept of perioperative interventions for ASD, specifically drawing upon the themes of enhanced communication with caregivers and incorporating this acquired knowledge into individualised care plans. Despite the case-focused, hospital-specific nature of much of the research and the methodological weaknesses noted, the overwhelmingly positive tone from efforts directed at improved communications with caregivers and individualisation of perioperative care supports further investigation. 
Table 1 Selected studies/case reports summarised by study characteristics, methods and outcomes

\begin{tabular}{|c|c|c|c|c|c|}
\hline $\begin{array}{l}\text { First author } \\
\text { (year) }\end{array}$ & $\begin{array}{l}\text { Participants } \\
\text { (N) }\end{array}$ & $\begin{array}{l}\text { Age } \\
\text { (years) }\end{array}$ & Country & Study design/assessment type & $\begin{array}{l}\text { Outcomes and recommendations for perioperative } \\
\text { interventions }\end{array}$ \\
\hline $\begin{array}{l}\text { Thompson } \\
(2014)^{8}\end{array}$ & 43 & $2-16$ & USA & $\mathrm{ABA}$ & $\begin{array}{l}\text { Better communication of individual needs enhances staff } \\
\text { comfort, parent satisfaction and patient experience }\end{array}$ \\
\hline $\begin{array}{l}\text { Christiansen } \\
(2005)^{9}\end{array}$ & 1 (case report) & 13 & Australia & Case report & $\begin{array}{l}\text { Emphasise parental involvement, review of anaesthesia } \\
\text { techniques with focus on 'combative' and } \\
\text { 'uncooperative' child }\end{array}$ \\
\hline Seid $(1997)^{10}$ & 2 (case reports) & & USA & Best practices/case report & $\begin{array}{l}\text { (1) Parent/caregiver to be used as expert/consultant on } \\
\text { child's idiosyncrasies; (2) pre-teaching adjusted to } \\
\text { cognitive capacity of patient; (3) separation from carer } \\
\text { minimised; (4) disruption of patient's routine minimised; } \\
\text { (5) distractors/treasured objects used to decrease anxiety }\end{array}$ \\
\hline $\begin{array}{l}\text { Lindberg } \\
(2012)^{11}\end{array}$ & 12 Parents & & $\begin{array}{l}\text { Sweden, } \\
\text { Finland }\end{array}$ & $\begin{array}{l}\text { Case report-parent report of child's } \\
\text { induction in the presence and absence of } \\
\text { dialogue }\end{array}$ & $\begin{array}{l}\text { Continuity in the perioperative dialogue with parents, } \\
\text { increased familiarity with staff (photo of nurse). } \\
\text { Eloquent illustration of historical tone of attributing } \\
\text { despair to procedure-related child/parent anxiety vs } \\
\text { suffering caused by care }\end{array}$ \\
\hline $\begin{array}{l}\text { Ambardekar } \\
(2013)^{12}\end{array}$ & & & USA & $\begin{array}{l}\text { Case report of incorporating service animal in } \\
\text { perioperative care of } 12 \text { year old with ASD }\end{array}$ & $\begin{array}{l}\text { Extension of family-centred care to include service dogs } \\
\text { makes experience less stressful for patients/families. } \\
\text { Author proposed 'induction room' so that the dog would } \\
\text { not compromise the sterility of the surgical procedure }\end{array}$ \\
\hline $\begin{array}{l}\text { van der Walt } \\
(2001)^{13}\end{array}$ & $\begin{array}{l}59 \text { Patients, } 87 \\
\text { procedures }\end{array}$ & $\begin{array}{l}26 \text { months- } \\
17 \text { years }\end{array}$ & Australia & $\begin{array}{l}\text { Review/audit of } 59 \text { patients with ASD in a } \\
\text { total of } 87 \text { procedures requiring anaesthesia }\end{array}$ & $\begin{array}{l}\text { Advanced notification, involvement of parents in } \\
\text { planning periprocedural elements and from induction to } \\
\text { early in the recovery phase were all emphasised. Flexible } \\
\text { admission (decreased waiting time) and quiet room }\end{array}$ \\
\hline $\begin{array}{l}\text { Souders } \\
(2002)^{14}\end{array}$ & 62 & $3-8$ & USA & $\begin{array}{l}\text { Best practices review of nursing care for } \\
\text { participants in study of secretin effects on } 62 \\
\text { children with ASD by Levy et al and Coplan } \\
\text { et al (2001) }\end{array}$ & $\begin{array}{l}\text { Focused preparation with family before hospital. Children } \\
\text { with high-functioning ASD: modelling, choices, } \\
\text { distraction and conversation and token systems. Children } \\
\text { with mild-moderate ASD: imitation, shaping, choices } \\
\text { and distraction. Children with severe ASD: combination } \\
\text { of high-probability requests/low-probability requests, } \\
\text { distraction with singing, counting and holding }\end{array}$ \\
\hline $\begin{array}{l}\text { Scarpinato } \\
(2010)^{15}\end{array}$ & 9 Cases & & USA & Clinical case studies and literature review & $\begin{array}{l}\text { Comprehensive initial assessment informing strategies } \\
\text { for intervention at Children's Hospital of Philadelphia. } \\
\text { Focused preplanning with family and individualisation. } \\
\text { Instructive neurosurgical case }\end{array}$ \\
\hline $\begin{array}{l}\text { Nelson } \\
(2009)^{16}\end{array}$ & & & USA & Perioperative guidelines & $\begin{array}{l}\text { Review of key strategies for perioperative nursing care, } \\
\text { organised by phase }\end{array}$ \\
\hline $\begin{array}{l}\text { Davignon } \\
(2014)^{17}\end{array}$ & $\begin{array}{l}20 \text { Mothers } \\
20 \text { Providers }\end{array}$ & & & Semistructured interviews & $\begin{array}{l}\text { Advanced preparation and communication with families } \\
\text { informing individualised modifications in patient flow } \\
\text { and clinical environment improve care }\end{array}$ \\
\hline $\begin{array}{l}\text { Vlassakova } \\
(2016)^{18}\end{array}$ & & & & Review & $\begin{array}{l}\text { Familiarity with patient's specific behavioural } \\
\text { characteristics and efforts to alleviate stress are keys to } \\
\text { smooth perioperative course }\end{array}$ \\
\hline
\end{tabular}

ASD, autism spectrum disorder.

Themes distilled from the perioperative management practices for ASD surveyed in this review contribute some order to the chaos that typically surrounds these patients. Further research directed at best practices will help to foster direct, useable platforms for care. These will be built from in-depth interviews of the significant people in these patients' lives that focus on root causes of problem behaviours. Efforts to increase predictability for the ASD surgical patient with Social Stories or desensitisation facilitated by pre-surgery hospital visits both appear promising interventions that may also help. In general, providers and patients will benefit by resisting unreflected routines and tapping the wealth of parent/caregiver knowledge about their child's idiosyncrasies.

Acknowledgements The authors gratefully acknowledge Briar Dechant, Jessie Lucas and Oren Gordon for their participation in the production of this article. We also acknowledge the guidance provided by paediatric anaesthesiologist, Alan Bielsky and paediatric surgeon, Frederick Karrer.

Competing interests None declared.
Provenance and peer review Not commissioned; externally peer reviewed.

\section{REFERENCES}

1 American Psychiatric Association. Diagnostic and statistical manual of mental disorders (DSM-5). American Psychiatric Publisher, 2013.

2 Siegel M, Gabriels RL. Psychiatric hospital treatment of children with autism and serious behavioral disturbance. Child Adolesc Psychiatr Clin N Am 2014;23:125-42.

3 Volkmar F, Siegel M, Woodbury-Smith $M$, et al. Practice parameter for the assessment and treatment of children and adolescents with autism spectrum disorder. J Am Acad Child Adolesc Psychiatry 2014;53:237-57.

4 Kopecky K, Broder-Fingert S, lannuzzi D, et al. The needs of hospitalized patients with autism spectrum disorders: a parent survey. Clin Pediatr (Phila) 2013;52:652-60.

5 Mills B. In the doctor's office: a parent perspective. Pediatr Clin North Am 2012;59:13-18

6 Gabriels RL. Adolescent transition to adulthood and vocational issues. In: Amaral DG, Dawson G, Geschwind DH, eds. Autism spectrum disorders. New York, NY: Oxford University Press, 2011:1167-81.

7 McGonigle JJ, Migyanka JM, Glor-Scheib SJ, et al. Development and evaluation of educational materials for pre-hospital and emergency department personnel on the 
care of patients with autism spectrum disorder. J Autism Dev Disord 2014;44:1252-9.

8 Thompson DG, Tielsch-Goddard A. Improving management of patients with autism spectrum disorder having scheduled surgery: optimizing practice. J Pediatr Health Care 2014;28:394-403.

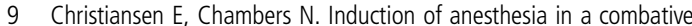
child; management and issues. Paediatr Anaesth 2005;

15:421-5.

10 Seid M, Sherman M, Seid AB. Perioperative psychosocial interventions for autistic children undergoing ENT surgery. Int I Pediatr Otorhinolaryngol 1997;40:107-13.

11 Lindberg S, von Post I, Eriksson K. The experiences of parents of children with severe autism in connection with their children's anaesthetics, in the presence and absence of the perioperative dialogue: a hermeneutic study. Scand I Caring Sci 2012;26:627-34.
12 Ambardekar AP, Litman RS, Schwartz AJ. "Stay, give me your paw": the benefits of family-centered care. Anesth Analg 2013;116:1314-16.

13 van der Walt J, Moran C. An audit of perioperative management of autistic children. Paediatr Anaesth 2001;11:401-8.

14 Souders MC, Freeman KG, DePaul D, et al. Caring for children and adolescents with autism who require challenging procedures. Pediatr Nurs 2002;28:555-62.

15 Scarpinato N, Bradley J, Kurbjun K, et al. Caring for the child with an autism spectrum disorder in the acute care setting. J Spec Pediatr Nurs 2010;15:244-54

16 Nelson D, Amplo K. Care of the autistic patient in the perioperative area. AORN J 2009;89:391-7.

17 Davignon MN, Friedlaender E, Cronholm PF. Parent and provider perspectives on procedural care for children with autism spectrum disorders. J Dev Behav Pediatr 2014:35:207-15.

18 Vlassakova BG, Emmanouil DE. Perioperative considerations in children with autism spectrum disorder. Curr Opin Anaesthesiol 2016;29:359-66. 